\begin{tabular}{lll} 
Abstract P021 Table & \multicolumn{2}{l}{ Results: referral numbers } \\
\hline & 2019 & 2020 \\
\hline Jan-March & 17 & 16 \\
April-June & 22 & 11 \\
July-Sept & 17 & 25 \\
Oct-Dec & 26 & 28 \\
\hline
\end{tabular}

36 patients $(45 \%)$ were offered surgical (6) or radiological (30) therapy in 2020 compared to 32 in 2019.

Similar radiological therapy was recommended both years. The main difference in surgical treatment was that only 3 resections were undertaken in 2020, compared to 7 in 2019. In 2020, 2 patients were referred for transplant assessment in November and December 2020 (none referred earlier in year), with 3 referred in 2019.

Palliative care was recommended for 44 (55\%) patients in 2020 , which was similar to $48(58 \%)$ of patients in 2019 . In 2020, 10 patients were referred for systemic therapy (17 in 2019). 2 patients received sorafenib compared to 7 the previous year.

Of those referred for palliative care, 17 had treatable disease but the patient was not fit for treatment while another 17 had extensive/metastatic disease and so were recommended for supportive care.

Conclusion COVID 19 may have had an impact on referral patterns and HCC screening programs in 2020. However, the total number of HCC cases referred to the regional HPB MDM in 2020 was as expected and the treatment options recommended were very similar to 2019. The only substantial difference was a reduction in the number of patients who were commenced on systemic therapy.

\section{P022 CLINICAL PRESENTATION AND OUTCOMES OF WILSON'S DISEASE: A SINGLE CENTRE COHORT STUDY}

Akhilesh Mulay*, James Dooley, Emmanouil Tsochatzis. UCL Institute for Liver and Digestive Health, Royal Free Hospital and UCL, London, UK

\subsection{6/gutjnl-2021-BASL.31}

Introduction Wilson's Disease is a disorder of copper homeostasis leading to hepatic and neuropsychiatric disease. Clinical data on these patients are limited due to low disease frequency. This report explores the presentation, management and outcomes of a large cohort of patients with Wilson's disease including pregnancy outcomes.

Methods A retrospective analysis was performed on 63 patients with Wilson's disease managed at the Royal Free Hospital, London, between 2012 and 2021. Clinical data were collected on presenting features, investigations, treatments and outcomes.

Results The mean age at diagnosis was 20 years (range 7-54 years) with 38 patients presenting due to liver involvement, 13 due to neuropsychiatric disease, 4 with both and 8 due to family screening. Of those presenting with liver involvement, $43 \%$ had asymptomatic liver disease with incidentally identified abnormal liver blood tests. The mean delay between onset of symptoms and diagnosis was 2 years (max. 8 years). During disease management, 31 patients received one type of drug treatment while 32 received more than one. In total, 48 patients received Penicillamine; of these, 11 were noncompliant to treatment, 16 experienced adverse effects and 21 stopped treatment. The most common adverse effect was skin changes (38\% of patients). In patients who stopped Penicillamine, $67 \%$ did so due to adverse effects. Trientine was given to 25 patients ( 1 noncompliant, 2 with adverse effects and 6 stopped treatment) while Zinc was given to 22 patients (4 noncompliant, 3 with adverse effects and 8 stopped treatment). At latest follow-up (mean 10.2 years), 92\% of patients with symptomatic liver disease, $87 \%$ with neurological disease and $89 \%$ with psychiatric symptoms had clinically improved or were stable. In patients with no psychiatric involvement at diagnosis, 26\% developed new psychiatric symptoms. Four patients underwent liver transplant, 3 due to decompensated cirrhosis and 1 due to acute liver failure. There were 3 deaths, of which 2 were due to liver-related complications. Seven patients had 12 pregnancies; 10 were on Penicillamine and 2 were on Zinc therapy throughout the pregnancy. Of those pregnancies on Penicillamine, 8 were normal, 1 was a miscarriage and 1 was a termination. Both pregnancies on Zinc were normal.

Conclusions The majority of patients with Wilson's disease who present primarily with liver involvement are asymptomatic with incidentally identified abnormal liver blood tests. New psychiatric symptoms commonly develop after diagnosis and may warrant more proactive psychological input. Penicillamine and Zinc appear to be safe in pregnancy.

\section{P023 THE EFFECTIVE USE OF BEZAFIBRATE AS A SECOND LINE THERAPY FOR PRIMARY BILIARY CHOLANGITIS}

Adam Howden*, Talal Valliani. North Bristol NHS Trust Liver Unit, UK

\subsection{6/gutjnl-2021-BASL.32}

Background Bezafibrate, a peroxisome proliferator-activated receptor alpha agonist, is not often used in the UK for treatment of PBC (primary biliary cholangitis) in patients who don't respond to ursodeoxycholic acid (UDCA) because it is an unlicensed therapy for this condition. In our liver unit we have used this treatment since 2014 .

Methods This retrospective case series included all patients who were commenced on bezafibrate from January 2014 to March 2020. 25 patients were listed from our pharmacy database and notes reviewed using a data collection tool. Biochemical and clinical outcomes were recorded over a 12 month period following initiation of bezafibrate in combination with UDCA.

10 patients were excluded due to either: non-compliance; a revised diagnosis of an overlap syndrome or primary sclerosing cholangitis; less than 12 months duration of bezafibrate therapy; lost to follow-up; not fulfilling PBC diagnostic criteria as per The British Society Gastroenterology/UK-PBC management guidelines. ${ }^{1}$

An inadequate response to UDCA therapy was defined by the 'Toronto' biochemical stratification as a serum alkaline phosphatase (ALP) level above $1.67 \mathrm{x}$ upper limit of normal (normal range $30-130 \mathrm{U} / \mathrm{L}$ ). This value is $217 \mathrm{U} / \mathrm{L}$ in our unit.

Results The 15 patients were all female with a median age at diagnosis of $49.67 \%$ were positive for anti-mitochondrial antibody or PBC specific anti-nuclear antibody. 33\% had consistent liver histology and the median ALP was $477 \mathrm{U} / \mathrm{L}$ at 